Revue des patrimoines

\title{
Devenir archiviste : la construction de la professionnalité en formation initiale universitaire
}

Becoming an archivist, the development of professionality in a university

training programme

Bénédicte Grailles

\section{(2) OpenEdition}

\section{Journals}

Édition électronique

URL : http://journals.openedition.org/insitu/13697

DOI : 10.4000/insitu. 13697

ISSN : $1630-7305$

Éditeur

Ministère de la culture

Référence électronique

Bénédicte Grailles, « Devenir archiviste : la construction de la professionnalité en formation initiale universitaire », In Situ [En ligne], 30 | 2016, mis en ligne le 03 octobre 2016, consulté le 19 avril 2019 URL : http://journals.openedition.org/insitu/13697 ; DOI : 10.4000/insitu.13697

Ce document a été généré automatiquement le 19 avril 2019.

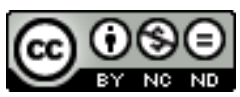

In Situ Revues des patrimoines est mis à disposition selon les termes de la licence Creative Commons Attribution - Pas d'Utilisation Commerciale - Pas de Modification 4.0 International. 


\section{Devenir archiviste : la construction de la professionnalité en formation initiale universitaire}

Becoming an archivist, the development of professionality in a university

training programme

Bénédicte Grailles

1 C'est à partir des années 1970 que les formations archivistiques universitaires commencent à se développer en France. Parallèlement à leur progressive implantation, c'est la définition même de la profession d'archiviste qui est soumise à interrogation. Être archiviste, c'est pendant longtemps être archiviste paléographe. À l'aube des années 1970, hors direction des Archives de France et Archives nationales, celui qu'on désigne comme étant « l'archiviste » est le chef d'un service départemental, voire municipal, aux effectifs la plupart du temps restreints et dont les agents n'ont reçu souvent aucune formation spécifique autre que celle du terrain. Cette définition est, non sans débats, peu à peu élargie à d'autres cadres d'emplois et de fonctions, dans un contexte favorisé par la décentralisation et la création de la filière culturelle dans la fonction publique territoriale. La désignation comme archiviste ne concerne plus seulement le conservateur du patrimoine mais englobe tous ceux qui exercent effectivement le métier d'archiviste. L'affectation dans un grade de la fonction publique n'est progressivement plus le critère opérant. Elle cède le pas au profit de critères de missions, plus inclusifs, même si un flou demeure sur la définition même du ou des métiers d'archiviste. Au passage, se produit un glissement de l'érudition à la technicité, de l'histoire à l'archivistique. Les formations universitaires ont accompagné et favorisé cette évolution. L'objet de cet article n'est pas de faire l'histoire des programmes universitaires mais plutôt de proposer une réflexion sur les modalités de construction de l'identité professionnelle de l'archiviste aujourd'hui dans le cadre précis de la formation initiale. Pour ce faire, nous croiserons une revue de littérature avec plusieurs enquêtes par voie de questionnaire menées entre 2013 et 2015 dans le cadre de mémoires ou programmes de recherche, ainsi que des enquêtes 
d'évaluation des enseignements et les retours des tuteurs de stage concernant les étudiants de la formation de l'université d'Angers ${ }^{1}$. Nous nous concentrerons sur la question de la formation initiale, car elle constitue un point d'observation idéal sur la construction de l'identité professionnelle de l'archiviste ${ }^{2}$.

\section{L'affirmation des diplômes universitaires : une nouvelle définition du métier ?}

2 La France et le monde francophone se sont ouverts tardivement à la formalisation des savoirs archivistiques. Or l'existence de formations fait partie, comme le regroupement en association professionnelle, l'élaboration d'un code de déontologie ou la tentative de réglementer l'accès à une activité, des stratégies d'un groupe professionnel pour assurer sa reconnaissance sociale ${ }^{3}$.

\section{Des débuts laborieux}

3 La formation aux métiers des archives a été jusque dans les années 1970-1980 en France et dans le monde francophone envisagée comme une spécialisation à l'issue d'une formation d'historien. En 1951, le stage technique international d'archives, créé par le directeur des Archives de France, Charles Samaran, "pour initier aux méthodes archivistiques françaises et familiariser avec les incomparables richesses de nos dépôts les archivistes, historiens et étudiants étrangers " ${ }^{4}$ avait répondu à sa manière à des besoins de formation dédiée. Stage d'application des archivistes paléographes qui se destinent à la conservation en archives jusqu'en 1990, la formation, qui alterne interventions d'archivistes et stage de terrain, consacre une part importante à la doctrine ${ }^{5}$. En 1970 paraît le Manuel d'archivistique. Force est de constater que ce premier ouvrage de référence en français est publié tardivement. Les manuels en anglais paraissent pour l'un, celui de Hilary Jenkinson au Royaume-Uni, en 1922 et pour l'autre, celui de Theodore Schellenberg aux États-Unis, en $1956^{6}$. Parallèlement, les formations universitaires spécialisées francophones accusent un certain retard par rapport par exemple aux États-Unis où les premiers certificats archivistiques universitaires se développent dès les années cinquante ${ }^{7}$. Elles émergent timidement à la fin des années 1970 en France avec la création d'un diplôme à l'université de Mulhouse, suivie des universités de Lyon et Angers. Elles commencent dans les années 1980 au Québec ${ }^{8}$. En Belgique, on constate aujourd'hui encore une formation à deux vitesses, bien organisée côté flamand, encore timide côté wallon'. En France, la formation est longtemps restreinte aux cadres, conçue comme empirique - on apprend sur le terrain - ou offerte aux archivistes praticiens dans un contexte de formation continue. Dans la séparation disciplinaire classiquement à l'œuvre dans le domaine des archives, elle penche du côté de l'histoire plus que des sciences de l'information ${ }^{10}$.

\section{Des enjeux liés}

Or l'émergence de formations initiales induit des changements importants. D'évidence, elle oblige à une réflexion sur les connaissances, les savoir-faire et les savoir-être, donc à un renouvellement du périmètre et de la géométrie du métier d'archiviste. Leur existence a des conséquences sur le métier lui-même et sur sa reconnaissance sociale. L'appréhension du métier d'archiviste a profondément évolué en un quart de siècle au 
point qu'il n'existe plus un archiviste mais des archivistes, même si l'affirmation de l'unité du métier continue à prédominer en France. Pour autant le débat ne semble pas pouvoir se réduire uniquement à une opposition entre une "nécessaire érudition » historienne et des gestes techniques ou une « technicité » aux contours quelque peu flous ${ }^{11}$, derrière lesquels on peut aussi lire une hiérarchie des intérêts entre archives anciennes et archives contemporaines. L'existence de formations initiales universitaires pose d'autres enjeux et notamment celui du statut de l'archivistique. La perception de l'archivistique, comme science ou technique, est une question débattue en Europe depuis les années 1990, qui a été abordée en France lors de journées d'étude co-organisées en 2003 par l'École des chartes et l'Association des archivistes français ${ }^{12}$. L'identification de cette problématique dénote la volonté des différents acteurs du monde archivistique de revendiquer une forme d'autonomie et des ambitions plus fortes. Au-delà, c'est la question de l'affirmation disciplinaire qui se pose car «la différentiation disciplinaire n'est pas uniquement une affaire épistémologique ou de potentiel heuristique, c'est aussi une question d'identité professionnelle et de positionnement social » ${ }^{13}$. Patrice Marcilloux, professeur des universités en archivistique, plaide d'ailleurs pour un régime transitoire d'interdiscipline, permettant de dépasser l'opposition entre une archivistique science auxiliaire de l'histoire et une archivistique science de l'information ${ }^{14}$.

\section{La professionnalisation à l'université}

5 Comment et sur quelles approches réglementaires, méthodologiques et pédagogiques s'opère la professionnalisation à l'université ? L'université n'est pas le lieu unique de formation initiale mais elle a ses contraintes et ses caractéristiques propres. Par ailleurs, l'apparition et la généralisation d'une offre de formation dans un secteur d'activités donné n'est pas sans conséquence pour le secteur lui-même.

\section{L'offre de formation universitaire aujourd'hui}

6 L'offre de formation des universités est systématiquement découpée en fonction du triptyque licence, master, doctorat (LMD). Coexistent des formations courtes (licence professionnelle achevant le cycle licence et n'autorisant pas de droit une inscription en master) et des formations longues (master, doctorat). Les formations aux métiers des archives s'inscrivent pleinement dans cette graduation. Il existe des licences professionnelles, des masters ${ }^{15}$ et un doctorat d'archivistique que seule l'université d'Angers délivre avec cette mention spécifique ${ }^{16}$.

7 Les diplômes nationaux font l'objet d'une habilitation par voie d'arrêté ministériel après évaluation. Tous les cinq ans, chaque université négocie son offre de formation avec le ministère en charge de l'Enseignement supérieur et passe un contrat qui insère l'offre de formation dans un projet global de développement. Depuis 2007, l'État est assisté dans cette tâche par une agence d'évaluation dédiée (autorité administrative indépendante) : l'Agence d'évaluation de l'enseignement et de la recherche (AERES) devenu le Haut Conseil de l'évaluation de la recherche et de l'enseignement supérieur (HCERES). Les rapports sont publics et consultables sur le site Web de l'agence ${ }^{17}$.

8 On pourrait résumer l'esprit de la professionnalisation à l'université de la manière suivante: il s'agit de transformer des savoirs empiriques acquis par l'expérience en savoirs académiques évalués de manière formelle ${ }^{18}$. Il n'y a pas, ou ne devrait pas y avoir, 
d'opposition entre activité scientifique et formation professionnelle. Ainsi, «lorsqu'une fonction de formation professionnelle est revendiquée à l'université, elle n'en est cependant pas moins replacée dans un contexte de développement de l'esprit scientifique $»^{19}$. Une formation professionnalisée a donc pour vocation de répondre à des besoins spécifiques et identifiés d'un secteur professionnel tout en les conciliant avec les attentes du monde universitaire.

\section{L'identification des compétences}

Depuis quelques années, l'évolution générale de la doctrine, impulsée par le ministère, est de gommer les frontières entre formation professionnalisée et formation non professionnalisée. Autrement dit, toutes les formations à l'université ont vocation à être professionnalisées, en tout cas au niveau master et licence professionnelle ${ }^{20}$.

La démarche préconisée dans l'élaboration ou la révision des contenus pédagogiques est une démarche d'identification des compétences. Elle est rendue obligatoire par la présence d'une annexe descriptive au diplôme dont la raison d'être est de lister les compétences validées par le diplôme à l'attention des futurs employeurs et de les rattacher à des unités d'enseignement. Les compétences à identifier peuvent être des compétences dites génériques (maîtrise de l'anglais par exemple), transversales (argumenter, animer une réunion, réaliser une synthèse...) ou spécifiques c'est-à-dire liées à un métier (mettre en œuvre la description archivistique suivant les normes du Conseil international des archives, utiliser et mettre à jour des thesaurus, etc.). L'annexe descriptive doit distinguer les connaissances acquises des savoir-faire et des savoir-être, sachant que l'accent est mis sur les connaissances et les savoir-faire plus que sur les savoir-être dont l'évaluation pose de vraies difficultés.

11 L'acteur principal de la construction du contenu des enseignements est le responsable identifié au moment du portage de la maquette. C'est généralement le responsable pédagogique du diplôme, appelé parfois directeur du master ou directeur des études. À chaque renouvellement de maquette, il capitalise l'expérience de fonctionnement. Chaque université a une grande latitude dans les processus mis en place pour piloter les diplômes. La mise en place de conseils de perfectionnement, d'évaluations des formations par les étudiants devrait être incontournable ${ }^{21}$. S'y ajoute le processus d'auto-évaluation réglementaire transmis ensuite à l'HCERES. Le responsable peut également s'appuyer sur les évaluations que chaque tuteur transmet en fin de stage.

Les acteurs universitaires se saisissent diversement de ce cadre. La réflexion sur les compétences est plus ou moins poussée en fonction de différents facteurs : une pression plus ou moins importante de l'établissement, un intérêt plus ou moins vif des équipes pédagogiques, une connaissance plus ou moins précise du secteur professionnel, des outils plus ou moins développés. Peuvent être notamment mobilisés les référentiels professionnels, celui de l'Association des archivistes français ${ }^{22}$ ou encore le référentiel des emplois-types de la Recherche et de l'Enseignement supérieur, REFERENS ${ }^{23}$, mais aussi les fiches de poste types, complétées par un dépouillement des offres d'emploi. C'est donc l'identification de compétences précises - génériques ou spécifiques - qui doit logiquement amener à l'introduction dans la démarche de formation de modes d'évaluation concordants. La démarche d'analyse est en forme de va-et-vient entre des compétences et des savoirs repérés a priori et leur traduction effective sur le terrain. 
13 Ainsi, à Angers, ce sont plus de 80 compétences qui ont été identifiées au niveau de la spécialité de master, également réparties entre compétences génériques ou transversales et compétences spécifiques. Il faut souligner que beaucoup de compétences sont de fait transversales - analyser des besoins, appliquer une démarche d'évaluation, concevoir et gérer un projet, concevoir et organiser des actions d'information, préparer et animer des formations, pour citer les plus évidentes - et sont ensuite exemplarisées dans l'environnement spécifique qui est celui de la gestion des archives et des documents d'activité.

L'approche par compétences a pour vertu d'irriguer l'ensemble de la formation. Elle permet la correspondance entre l'organisation et le programme de la formation et la profession cible. Elle permet de formuler et de prioriser des objectifs de formation, aide à la conception de l'évaluation des apprentissages et formalise le contrat didactique ${ }^{24}$. C'est pour cette raison que, bien que les diplômes soient nationaux, ils ne sont pas pour autant identiques.

\section{Des processus légitimants}

15 Toutes les formations professionnalisées présentent des points communs dans leurs méthodes pédagogiques - stage obligatoire notamment - et leurs modes d'évaluation avec, en acmé, l'élaboration et la présentation d'un mémoire proposant la synthèse d'une situation professionnelle. Cette synthèse doit répondre à des exigences de type universitaire : distanciée, elle fait état de questionnements sur la situation elle-même, sur l'activité du stagiaire mais aussi sur les normes et les consensus professionnels. La délivrance du diplôme sanctionne non seulement «la nature et la légitimité des connaissances et des compétences » mais aussi « la nature et la légitimité des modalités de cette acquisition $»^{25}$.

16 Entre l'exercice d'une profession et la délivrance d'un diplôme universitaire correspondant s'opère un double mouvement de co-légitimation. La délivrance du diplôme légitime son titulaire, alors autorisé à revendiquer la position d'archiviste. Mais en même temps, l'existence d'une formation initiale longue professionnalise et légitime un métier puisqu'elle formalise des savoirs jusque-là empiriques ${ }^{26}$. On notera par ailleurs que l'existence d'un corpus de savoirs abstraits ou formels, pour reprendre la terminologie formulée par Léonie Hénaut dans l'environnement muséal ${ }^{27}$, est un levier important de valorisation d'un métier.

\section{Une professionnalité émergente}

17 Ainsi, l'existence de formations et l'attribution de diplômes contribuent à la définition du périmètre d'un métier et à sa reconnaissance sociale. Formation et milieu professionnel véhiculent une ou des identités professionnelles que les étudiants vont chercher à acquérir par un processus de professionnalisation. Comment s'opère l'assimilation de ce que les sociologues et les spécialistes de sciences de l'éducation nomment professionnalité? Derrière ce mot se cachent «la construction d'une identité professionnelle, l'incorporation de compétences et de gestes professionnels, ainsi que les aspects éthiques et déontologiques de la profession concernée ${ }^{28}$. La professionnalité ne recouvre pas que la capacité d'agir avec discernement dans une situation donnée de travail mais intègre aussi la capacité à donner du sens à une activité, à dialoguer avec les 
représentations, les normes, les valeurs qui se tissent autour de cette activité. C'est un processus continu qui permet d'affirmer un positionnement professionnel ${ }^{29}$.

\section{Des étudiants et de leurs représentations}

Les étudiants arrivent en formation avec leurs propres représentations. Ils ont des motivations précises et des idées préconçues avec lesquelles l'équipe pédagogique doit composer. Le responsable de formation en a des indices, notamment au moment de la procédure de recrutement (lettre de motivation, entretiens). Pour tenter de les objectiver, nous nous appuierons sur une enquête menée en 2013 par Sandy Guibert sur les archivistes et l'émotion, dans le cadre de son mémoire de master à l'université d'Angers ${ }^{30}$. Certaines questions permettent d'éclairer l'entrée en formation. À la question 66 « Pourquoi avez-vous voulu travailler dans les archives? », la réponse était libre. L'analyse lexicométrique des 86 réponses montre que près de $90 \%$ des réponses contiennent le terme « histoire ", alors que le mot «archives » n'est utilisé que par $35 \%$ des répondants, tout en étant le second terme le plus fréquemment utilisé, loin devant les suivants (fig. 1). L'usage du mot « document(s) » est également révélateur : il a été utilisé associé aux qualificatifs « anciens », « historiques », « vieux » et « public ». Dans la même enquête, la question 9 « Si vous deviez attribuer un adjectif au mot « archives », ce serait : ... » proposait de choisir un ou plusieurs qualificatifs dans une liste ouverte ${ }^{31}: 85 \%$ des répondants ont choisi l'adjectif « historiques ».

Figure 1

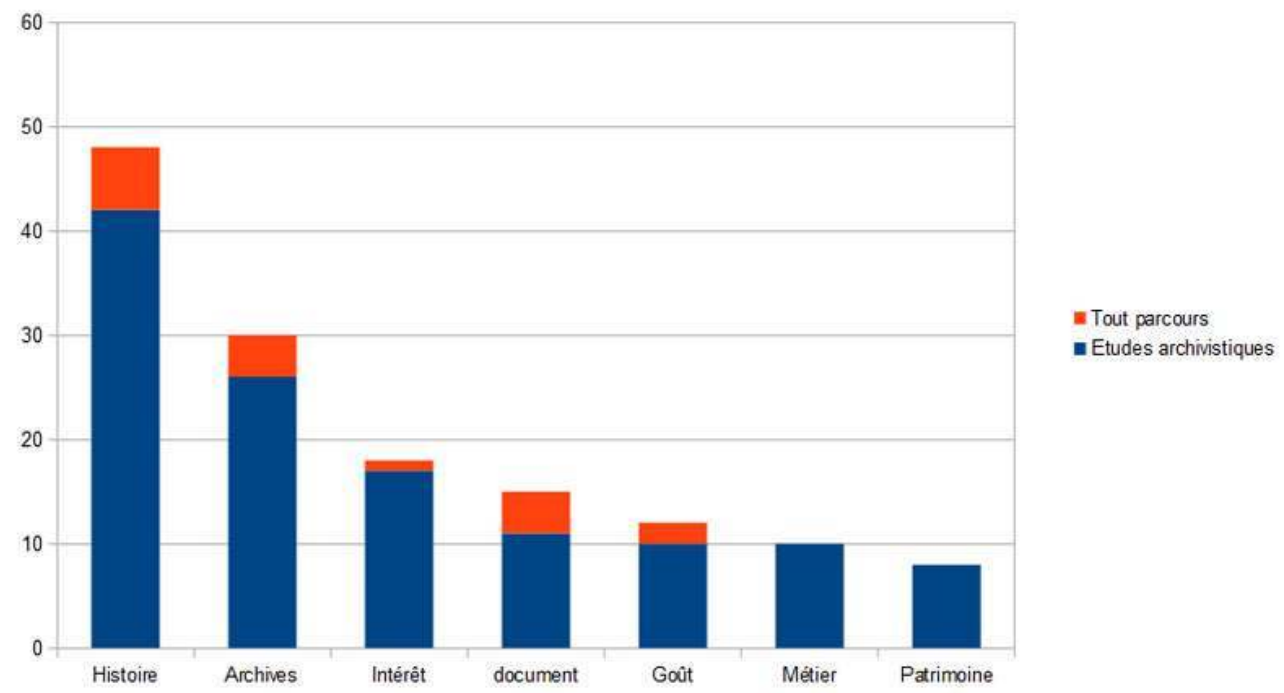

Analyse lexicométrique des réponses à la question « Pourquoi avez-vous voulu travailler dans les archives ? » (réponse libre) : termes les plus utilisés. Enquête «Archives et émotion » de Sandy Guibert (master université d'Angers) auprès d'archivistes et d'étudiants en archivistique, 2013.

19 Ces réponses dessinent assez précisément les représentations préalables des étudiants et leur force. Les étudiants issus de la filière histoire arrivent avec un point de vue focalisé sur les usages historiens des archives. Ils confondent parfois encore le métier d'historien et celui d'archiviste. Ils ont souvent une vision patrimoniale des archives, tournée vers la valorisation. Le niveau de recrutement du diplôme influe considérablement sur les profils des étudiants. Les étudiants qui postulent pour une entrée directe en deuxième année de 
master sont généralement issus d'un cursus en histoire. Plus on propose une spécialisation en archivistique tôt dans le cursus, plus les profils se diversifient. C'est le cas à l'entrée en première année de master, à condition que le master soit vraiment bien identifié comme spécialisé dès cette année ; c'est le cas aussi en licence professionnelle. Ainsi, pour l'entrée en première année de master à Angers en 2014, les candidatures provenaient de dix licences différentes, alors qu'à l'entrée en deuxième année, hors étudiants déjà inscrits l'année précédente dans le même master, on ne trouvait plus que trois provenances disciplinaires différentes.

Il faut aussi relever que les étudiants qui choisissent une formation en archives ont déjà réalisé un voire plusieurs stages (fig. 2). Ces stages ont joué un rôle déterminant puisqu'ils ont conforté un projet professionnel naissant. L'écrasante majorité de ces premiers stages se déroule dans le réseau des archives publiques, généralement dans des services à vocation patrimoniale, archives départementales ou communales. C'est donc sur la base de ces premières expériences que commence à se construire l'identité professionnelle des étudiants. Elles conditionnent fortement leur vision du métier, plutôt tournée vers les archives historiques.

Figure 2

I Combien de stage avez-vous effectué depuis le début de votre entrée dans l'enseignement supérieur?

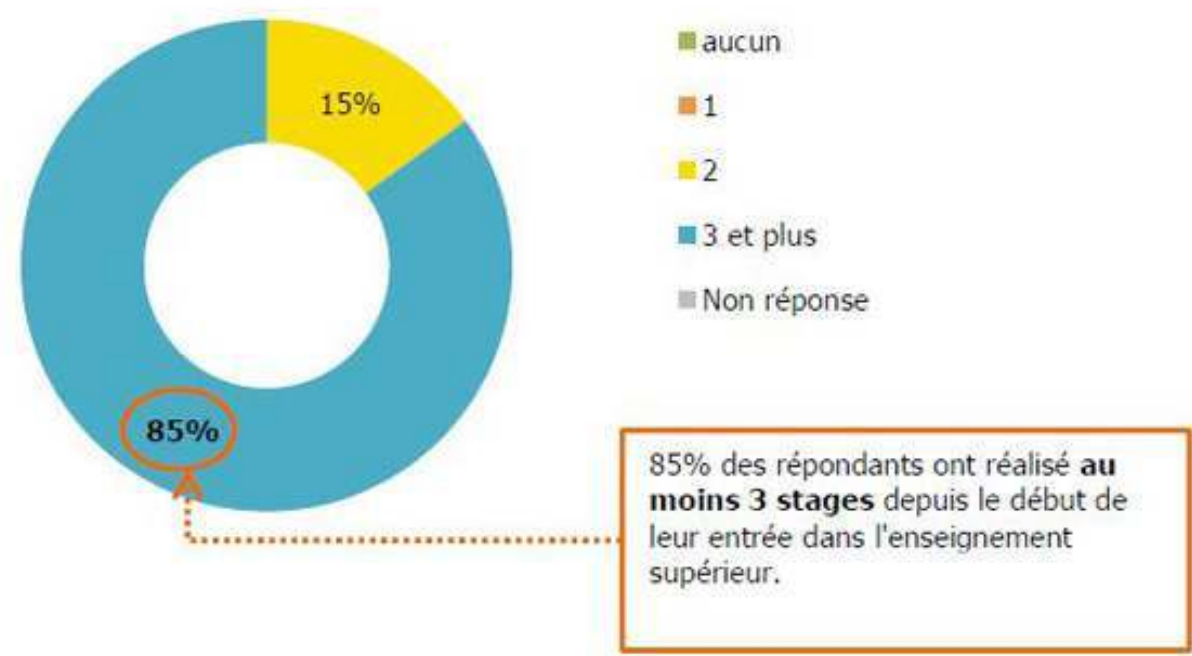

Nombre de stages effectués depuis l'entrée dans l'enseignement supérieur avant l'entrée en deuxième année de master. Enquête de l'université d'Angers auprès des étudiants de deuxième année de master Métiers des archives de l'établissement, promotion 2013-2014.

(c) Université d'Angers.

\section{Un travail de conversion}

21 Le rôle d'une formation professionnalisée est de permettre progressivement aux étudiants de faire la distinction entre ces idées préconçues et la réalité du travail, la diversité des situations et des environnements, donc de faire le deuil d'une vision idéalisée au profit d'une vision réaliste. C'est ce que nous sommes tentée d'appeler un travail de conversion : il faut devenir archiviste. 

70 programmes universitaires ${ }^{32}$, avait noté «que les cours magistraux n'occupent en moyenne que $50 \%$ du temps d'enseignement alors que l'autre moitié se compose essentiellement d'activités de nature pratique (laboratoires, travaux pratiques, travaux dirigés) $»^{33}$. Si on peut s'interroger sur ce rapport apparemment équilibré entre cours magistraux et travaux dirigés, sur des catégories qui ont disparu aujourd'hui au profit d'autres, à savoir enseignements transversaux, disciplinaires et professionnels, et sur la difficulté à classer les enseignements d'archivistique, tout à la fois disciplinaires et professionnels, on constate que les outils pédagogiques de mise en lien entre réalités de terrain et apports théoriques ou réflexifs se sont affinés au cours des années. Dans la construction de la professionnalité et la conversion des étudiants en archivistes, quatre points méritent une vigilance particulière car ils favorisent ou accentuent la mise en mouvement ${ }^{34}$.

L'identification des compétences est l'un d'entre eux. Outre son utilité dans la construction d'un programme, elle permet de fixer des objectifs clairs en termes d'acquisitions. C'est donc un moyen de partager, entre intervenants et avec les étudiants, la philosophie de la formation, de constituer une communauté. Parce qu'elle génère du collectif, il est possible de s'appuyer sur elle pour mettre en œuvre des méthodes pédagogiques appropriées et parfois originales permettant de développer des formes d'évaluation différentes de celles habituellement à l'œuvre pour les savoirs disciplinaires traditionnels.

modalités pédagogiques concourent efficacement à la construction professionnelle et permettent à cette communauté de prendre corps. Ainsi, le travail en mode projet et en groupes nécessite entre les étudiants mêmes, entre les étudiants et les partenaires du projet, entre les étudiants et l'enseignant-référent du projet, l'élaboration de compromis, la prise en compte empathique des besoins, des exigences et possibilités des uns et des autres, et implique des négociations et des ajustements constants. Les connaissances, savoir-faire et savoir-être acquis au sein d'un groupe de projet sont partagés avec l'ensemble de la promotion et des interactions se mettent en place entre les groupes tout au long de l'expérience permettant de dégager collectivement de ces différentes situations professionnelles des savoirs universitaires.

prise en compte progressive des réalités du métier passe aussi par l'intégration des attendus du marché du travail. Une réflexion sur le curriculum vitae - sur ses propres compétences $^{35}$ - et sur les offres d'emploi est un levier très utile.

stage est bien évidemment un moment central ${ }^{36}$ parce qu'il permet d'éprouver un grand nombre de connaissances théoriques et de les confronter aux pratiques professionnelles d'un environnement donné, à des situations de travail: "A failed experience can be so confusing and frustrating as to call into question everything the student has learned in class and even his or her choice of careers. $\wedge^{37}$ Jean-Yves Rousseau relève néanmoins que si le stage est une période privilégiée pour l'intégration des pratiques et le développement d'un regard critique, il «ne contribue pas assez à dégager un savoir stratégique ou savoir pour l'action $»^{38}$. Comme pour les projets, il faut rebondir sur l'expérience pour en dégager des savoirs universitaires.

27 Ces différents éléments font progressivement évoluer l'étudiant qui, après quelques semaines, est en capacité de repérer des besoins d'apprentissage qu'il n'anticipait pas à 
son entrée en formation. C'est le signe qu'il a changé de posture. Il ne se considère plus comme étudiant mais comme apprenti professionnel ${ }^{39}$.

Dans cette évolution propre à chacun mais dans une dynamique collective, la responsable de la formation que nous sommes formule les constats suivants. Plus les étudiants sont ancrés dans un vécu disciplinaire commun, plus ils ont tendance à s'y conformer. Il est donc plus facile de faire évoluer un groupe avec des expériences et des vécus différents. Par ailleurs, il faut laisser du temps aux étudiants pour qu'ils puissent faire ce travail sur eux-mêmes. Il y a donc une corrélation directe entre la mixité des origines et la capacité à faire bouger les lignes, ainsi qu'entre la durée de la formation et la possibilité de faire accepter des visions du métier plus éloignées des idées préconçues et des représentations sociales communément partagées.

\section{Une co-construction}

Dans une formation, le mécanisme d'apprentissage n'est pas uniquement vertical et descendant; il ne s'agit pas uniquement d'écouter et d'engranger l'évidence de la parole enseignante. D'autres acteurs participent à l'élaboration de la construction de l'identité professionnelle: les intervenants professionnels extérieurs bien sûr, les tuteurs ou tutrices de stage bien évidemment, mais aussi les pairs, c'est-à-dire les autres étudiants. Entre tous les membres de la communauté se nouent des interactions qui sont des moteurs d'apprentissage très efficaces.

Le tuteur de stage joue un rôle symbolique fort. Michel Champagne a bien montré ses responsabilités et ses limites ${ }^{40}$, en décrivant le milieu de stage non comme un lieu de "croissance personnelle» mais un lieu de "croissance professionnelle». Il a relevé également que «le stage est pour beaucoup de milieux une contribution consciente au développement de la communauté archivistique $»^{41}$. Les intervenants extérieurs participent à la médiation entre le métier ou le segment de métier qu'ils incarnent et les étudiants. On pourrait qualifier ces différents rôles d'« intercession» - pour rester dans le vocabulaire de la conversion.

L'enseignant responsable de formation, en tant que référent universitaire, assume une fonction pivot dans ce processus d'apprentissage. Entre ce que l'étudiant imagine du métier et l'image qui lui est renvoyée par les professionnels, les tuteurs lors des stages ou ses propres camarades, ou les débats qui existent au sein de la profession et dont il prend conscience, il y a des décalages qui génèrent des tensions identitaires. Le référent universitaire intervient pour faire dialoguer ces différentes visions afin qu'émerge un positionnement à la fois professionnel et distancié.

D'une manière générale, la légitimité de tous les formateurs est renforcée par leur expérience professionnelle et le fait de partager avec les étudiants une identité professionnelle donnée.

Enfin, il ne faut pas négliger ce qui se passe à l'intérieur du groupe, entre pairs étudiants. Dans ces rapports-là aussi, par le jeu des ajustements et des interactions, par les postures mobilisées et la dynamique identitaire, chaque étudiant apprend à devenir professionnel. 


\section{Acquérir les habitus de la profession}

34 qui lui sont propres dans une certaine dynamique. Le contenu seul ne suffit pas à influer sur les représentations et sur les besoins d'apprentissage. C'est une alchimie complexe qui repose aussi sur la capacité de l'étudiant à se mettre en mouvement et à se sentir légitime. On peut tenter de mesurer ce processus en évaluant l'adhésion que l'intéressé construit vis-à-vis de sa formation et de son futur métier. La période de formation contribue à l'incorporation des habitus professionnels ${ }^{42}$.

Comme dans tout processus d'apprentissage, l'étudiant construit son soi professionnel en adhérant progressivement à la formation qu'il suit et ce, sous différents angles ${ }^{43}$. Il lui faut notamment accepter et jouer le jeu des méthodes pédagogiques proposées, être capable d'identifier les besoins d'apprentissage et de les corréler avec les méthodes pédagogiques. L'étudiant a aussi besoin de la reconnaissance du milieu professionnel : il se sentira conforté s'il déduit, lors des interactions avec les professionnels, que ceux-ci connaissent la formation et ont un jugement positif sur celle-ci, ce d'autant que le niveau d'investissement et d'implication personnels qui leur est demandé est perçu comme exigeant, voire très exigeant.

Au-delà de la question de la formation même, c'est la construction de l'identité professionnelle qui se joue. Celle-ci est difficile à mesurer. Nous pouvons tenter néanmoins de l'appréhender de manière déductive et comparative à travers les résultats d'une enquête menée en 2015, dans le cadre d'un projet collectif, par un groupe d'étudiants de deuxième année de master de l'université d'Angers ${ }^{44}$. L'objectif de cette enquête était d'explorer la perception de l'identité numérique chez les étudiants se destinant à des métiers documentaires - métiers des archives, des bibliothèques, professeurs-documentalistes - et en cours de formation dans la même universitét ${ }^{45}$. Deux questions permettent indirectement de percevoir la manière dont les étudiants sont en train de construire leur identité professionnelle et la relation de conformité au milieu qu'ils ont l'ambition d'intégrer. La première question portait sur la possibilité de conserver à des fins historiques et scientifiques des données informatiques nominatives (fig. 3).

Figure 3

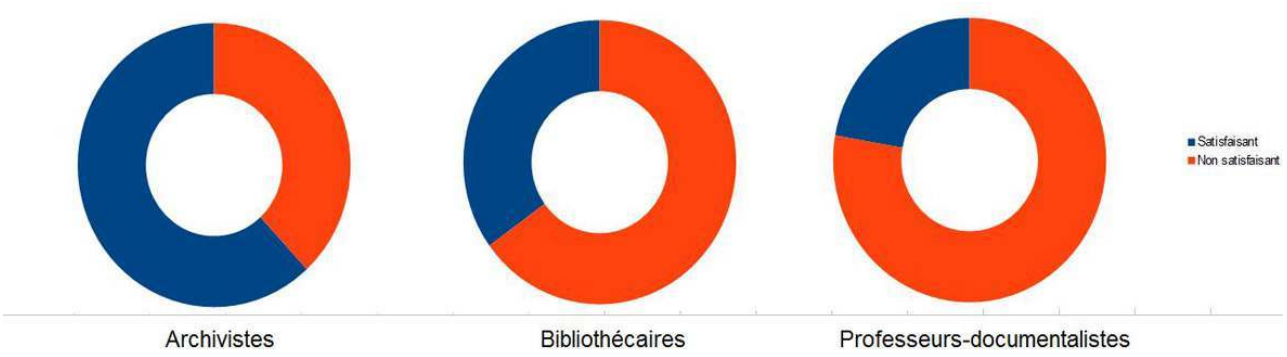

Réponses à la question fermée : « La loi permet aux services d'archives de conserver les données informatiques nominatives à des fins historiques et scientifiques. Trouvez-vous cela satisfaisant ou non satisfaisant? ». Enquête des étudiants du master Métiers des archives de l'université d'Angers promotion 2014-2015 auprès des étudiants de licence professionnelle ou masters d'Angers (archives, bibliothèques, professeurs-documentalistes), 2015. 
La seconde était formulée ainsi : «En 2013, la pétition de l'Association des archiviste français a recueilli 41000 signatures contre le projet de règlement européen sur l'anonymisation de toutes les données personnelles dont la finalité première serait devenue inutile. Qu'en pensez-vous?» (fig. 4). Dans les deux cas, on observe que les réponses sont très différentes en fonction du cursus choisi. De manière prévisible, les futurs archivistes sont très nettement plus concernés par la conservation à long terme des archives électroniques, y compris des données nominatives. Quant à la pétition \#Eudatap contre le projet de règlement européen sur les données personnelles ${ }^{46}$ qui a mobilisé très largement non seulement la communauté archivistique mais aussi ses partenaires généalogistes et historiens ${ }^{47}$, le contraste des réponses est saisissant et montre que, d'une part, tous les apprentis archivistes sont parfaitement au fait de ce débat entre droit à l'oubli et droit à la mémoire et que, d'autre part, leur positionnement est clairement assumé.

Figure 4

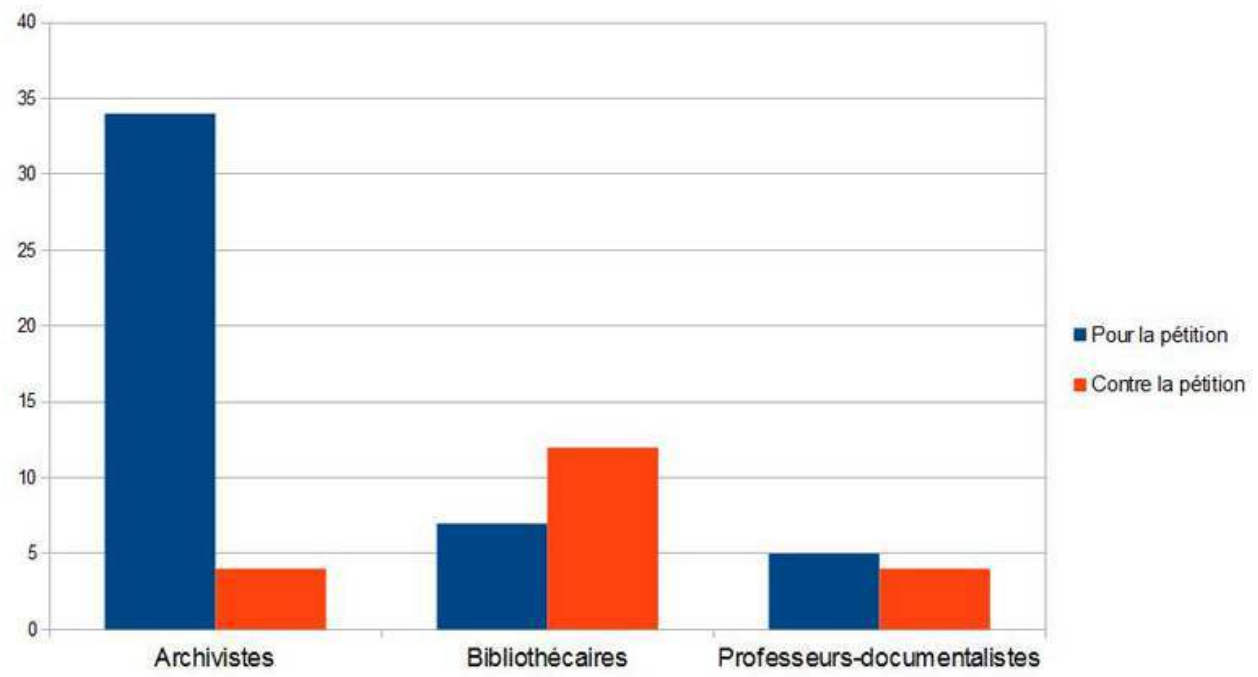

Réponses à la question fermée : «En 2013, la pétition de l'Association des archivistes français a recueilli 41000 signatures contre le projet de règlement européen sur l'anonymisation de toutes les données personnelles dont la finalité première serait devenue inutile. Qu'en pensez-vous ? ». Enquête des étudiants du master Métiers des archives de l'université d'Angers promotion 2014-2015 auprès des étudiants de licence professionnelle ou masters d'Angers (archives, bibliothèques, professeursdocumentalistes), 2015

À travers ces deux cas, on voit très nettement que les résultats sont connotés en fonction $\mathrm{du}$ cursus choisi. On pourrait être tenté d'imputer ce constat uniquement aux enseignements suivis, aux contenus pédagogiques, à une certaine philosophie de formation. Ce serait omettre une variable de taille : l'adhésion progressive à une culture professionnelle dominante.

\section{Conclusion}

Être archiviste, c'est être reconnu par les professionnels comme tel et c'est se reconnaître comme tel. Pour des étudiants souvent attirés par le volet historique du métier et qui découvrent qu'il ne s'agit que d'une facette parmi d'autres, l'émergence de la professionnalité est complexe. D'évidence, réussir une formation universitaire 
professionnalisée ne consiste pas seulement à engranger des connaissances et répéter des gestes techniques mais bien à construire une identité professionnelle en résonance avec le milieu visé. Devenir un professionnel des archives, ce n'est pas que reproduire des méthodes acquises mais c'est être en capacité de mobiliser connaissances, compétences, expériences - la sienne propre et celles transmises par d'autres -, savoir-être et savoirfaire d'une manière conforme aux pratiques, attendus et éthique de la profession, tout en projetant un regard analytique; c'est choisir une réponse à une situation en tenant compte des particularités de celle-ci, tout en sachant que d'autres réponses seraient possibles dans d'autres environnements et avec d'autres interactions.

L'acquisition de cette professionnalité passe conjointement, pour les étudiants en formation initiale, par la confrontation avec le terrain et par l'érection en savoirs formels capitalisés sur les bancs universitaires. Il en découle une forte interdépendance entre les différents acteurs, chacun concourant à sa place à la reconnaissance et la légitimation de la profession. Une formation universitaire ne peut se développer en décalage avec le milieu professionnel de référence, même si elle se doit d'introduire un point de vue distancié qui est la marque du monde universitaire et un gage d'adaptabilité pour les futurs professionnels, mais une profession a besoin de l'existence de diplômes reconnus et de formalisation des savoirs. De ce constat, il convient de conclure sur la nécessité d'un dialogue constant entre les différents acteurs, universitaires, professionnels de tous horizons, associations professionnelles et institutions de référence.

\section{NOTES}

1. - L'auteure du présent article est responsable pédagogique de la spécialité à finalité professionnelle et recherche du master Métiers des archives de l'université d'Angers. 2. - Sur la formation continue et pour un point de comparaison, GRAILLES, Bénédicte et MARCILLOUX, Patrice. « La formation continue des archivistes : une expérience universitaire ». Dans « La formation professionnelle : enjeux d'hier et d'aujourd'hui », La Gazette des archives, $\mathrm{n}^{\circ}$ 218, année 2010-2, p. 116-129.

3. - Sur la question du modèle professionnel, CHAPOULIE, Jean-Michel. « Sur l'analyse sociologique des groupes professionnels ». Revue française de sociologie, 1973, vol. 14, $\mathrm{n}^{\circ} 1$, p. 86-114.

4. - Lettre-circulaire aux attachés culturels en français et anglais pour l'année 1953-1954, Arch. nat., 20000381, art. 3.

5. - GRAILLES, Bénédicte. « Archivistiques canadienne et française : échanges, influences ou transferts? ». Dans BERGERON, Yves et FEREY, Vanessa (dir.). Archives et musées. Le théâtre du patrimoine (France-Canada). Paris : Éd. du CTHS, 2013, p. 47-66.

6. - JENKINSON, Hilary. A manual of archive administration. Oxford : Clarendon Press, 1922 (2 e éd., 1937, $3^{e}$ éd., 1965 par R.-H. Ellis) ; SCHELLENBERG, Theodore R. Modern archives. Principles and techniques. Chicago : University of Chicago Press, 1956 ( $2^{e}$ éd., 1957, nlle éd. 2003). 
7. - COX, Richard J., YAKEL, Elizabeth, WALLACE, David, BASTIAN, Jeannette A., MARSHALL, Jennifer. « Archival Education in North American Library and Information Science Schools ». The Libray Quaterly: Information, Community, Policy, vol. 71, $\mathrm{n}^{\circ} 2$, avril 2001, p. 141-194, p. 163.

8. - NAHUET, Robert. «L'AAQ [l'Association des archivistes du Québec] : la formation et le perfectionnement en archivistique ». Archives, vol. 39, nº 1, 2007-2008, p. 93-111.

9. - DEPORTEERE, Rolande. «Les archives en Belgique : une réalité éclatée ». Archives, vol. 34, nos 1-2, 2002-2003, p. 101-117.

10. - DELMAS, Bruno. « Origine et développement de l'enseignement de l'archivistique ». Archivum, vol. 34, p. 61-73. MARÉCHAL, Michel et EICHENLAUB, Jean-Luc. « La formation des archivistes en Europe ». Dans Les Archives françaises à la veille de l'intégration européenne : actes $\mathrm{du}$ XXXI ${ }^{e}$ congrès national des archivistes français, 23-26 octobre 1990. Paris : direction des Archives de France, Archives nationales, 1992, p. 107-129.

11. - RENÉ-BAZIN, Paule. « L'archiviste et ses partenaires : territoires propres, démarches convergentes ». Bulletin des bibliothèques de France, t. 5, p. 33-39. Marie-Françoise Limon propose de centrer la définition de la technicité archivistique autour de la problématique du traitement des documents. LIMON, Marie-Françoise. « La poursuite du développement et de la formation en archivistique ». Archives, vol. 31, n³ 3, 1999-2000, p. 49-60, p. 54. 12. - HOTTIN, Christian. « L'archivistique est-elle une science ? Réactions aux journées d'études organisées par l'École des chartes et l'Association des archivistes français à la Sorbonne (salle Louis-Liard) les 30 et 31 janvier 2003 ». Labyrinthe, 2003, n 16, p. 99-105. 13. - MARCILLOUX, Patrice. "L'archivistique à l'université : quel régime disciplinaire ?». La Gazette des archives, $\mathrm{n}^{\circ}$ 226, 2012-2, p. 298-311, p. 299.

14. - Ibid., p. 310.

15. - À l'université d'Angers, il existe des unités de pré-professionnalisation dès la deuxième année de licence, une licence professionnelle « Traitement et gestion des archives et des bibliothèques » comportant un tronc commun et des enseignements de spécialité (archives ou bibliothèque), un master « Métiers des archives » avec deux années complètement orientées vers les archives et un doctorat en archivistique. Notons que les licences professionnelles sont soumises à des procédures particulières d'habilitation. 16. - En effet, les écoles doctorales peuvent choisir les libellés des doctorats qu'elles délivrent. Seule l'école doctorale à laquelle est rattachée l'université d'Angers pour les sciences humaines a choisi d'inscrire l'archivistique officiellement dans ses matières.

17. - Voir le site : http://www.aeres-evaluation.fr/. Rubrique « Rapports ».

18. - POSTIAUX, Nadine et ROMAINVILLE, Marc. « Compétences et professionnalisation : La compétence asservit-elle l'Université au monde professionnel, la faisant ainsi renoncer à son idéal pédagogique? » [document électronique]. Éducation et Formation, $n^{\circ} 296,2011$, p. 45-55, p. 47, http://ute3.umh.ac.be/revues/index.php?revue=12\&page=3 [consulté le 05/08/2015].

19. - Ibid., 2011, p. 53.

20. - Un master recherche doit former au métier de chercheur, un master enseignement au métier d'enseignant, un master archives au métier d'archiviste.

21. - À Angers, il existe un conseil de perfectionnement rassemblant enseignants, étudiants, responsables scolarité et insertion professionnelle et professionnels du secteur. Ce dispositif est complété par des évaluations par les étudiants (anonymes et par voie d'enquêtes mais aussi collectives et orales).

22. - Association des archivistes français. La profession d'archiviste ou les métiers des archives. Référentiel métiers. Paris : Association des archivistes français, [2008]. 
23. - Voir le site : http://referens.univ-poitiers.fr/version/men/ [consulté le 27/09/2016]. 24. - POSTIAUX, Nadine, BOUILLARD, Philippe et ROMAINVILLE, Marc. « Référentiels de compétences à l'université : Usages, rôles et limites ». Recherche et Formation, $\mathrm{n}^{\circ}$ 64, 2010, p. 15-30.

25. - AYOUBE, Ranim. La construction de la professionnalité émergente en master professionnel: regards évaluatifs de stagiaires et de formateurs. Thèse de doctorat en sciences de l'éducation. Toulouse : université Toulouse-Le Mirail - Toulouse-II, 2013, p. 33.

26. - POSTIAUX, Nadine et ROMAINVILLE, Marc. « Compétences et professionnalisation... ». Art. cit., p. 47.

27. - HÉNAUT, Léonie. « Capacités d'observation et dynamique des groupes professionnels. La conservation des œuvres de musées ». Revue française de sociologie, vol. 52, 2011/1, p. 71-101.

28. - JORRO, Anne, BROCAL, Renée, POSTIAUX, Nadine. «Évaluer la professionnalité émergente en formation ». Dans ROMAINVILLE, Marc, GOASDOUÉ, Rémi, VANTOUROUT, Marc (dir.). Évaluation et enseignement supérieur. Bruxelles : De Boeck, 2013, p. 73-84, p. 76. 29. - Sur la notion de professionnalité et sur celle, dérivée, de professionnalité émergente, JORRO, Anne, DE KETELE, Jean-Marie. La professionnalité émergente : quelle reconnaissance? Bruxelles : De Boeck, 2011.

30. - GUIBERT, Sandy. Les archives, support d'émotions? Le point de vue des archivistes à l'ère du numérique, mémoire de recherche de master 1 Histoire, Document (métiers des archives) de l'université d'Angers, 2013 ; voir le site : http://alma.hypotheses.org/tag/ guibert-sandy [consulté le 27/09/2016] ; « Les archivistes et l'émotion ». HOTTIN, Christian, POTIN, Yann, SABLON DU CORAIL, Amable. Culture et Recherche, $\mathrm{n}^{\circ} 129$, hiver 2013-2014, p. 54 ; voir le site : http://www.culturecommunication.gouv.fr/var/ culture/storage/pub/culture_et_recherche_129/index.htm [consulté le 27/09/2016]. L'enquête comprenait 82 questions et s'adressait à des archivistes et à des étudiants en formation archivistique. Elle a été diffusée par le biais des réseaux sociaux et des forums spécialisés.

31. - 21 adjectifs étaient proposés. Le répondant pouvait choisir un item « autre » et apporter un qualificatif différent.

32. - Cette enquête effectuée dans le cadre d'un programme de recherche académique avait donné lieu à l'établissement d'une bibliographique internationale commentée sur la question de la formation des archivistes toujours utile bien qu'ancienne désormais ; voir le site : http://mapageweb.umontreal.ca/couturec/rapport/annexe9.htm [consulté le 27/09/2016].

33. - COUTURE, Carol. « La formation et la recherche en archivistique. Éléments révélateurs de l'état de développement de l'archivistique contemporaine ». Archives, vol. 33, n 2, 2001-2002, p. 21-51, p. 24.

34. - Sur la question des leviers pédagogiques, AYOUBE, Ranim. La construction de la professionnalité émergente... Op. cit.

35. - La question de l'élaboration et de la tenue de livrets de compétences par chaque étudiant est d'actualité dans le monde universitaire français. Les universités commencent à s'en emparer même si les modalités d'accompagnement restent pour l'essentiel à définir.

36. - La question du stage bénéficie d'une bibliographie abondante. Citons notamment ARÈS, Florence. «Le stage dans la formation universitaire ». Archives, 1989, vol. 20, n 3 , p. 53-60 ; BASTIAN, Jeannette A., WEBBER, Donna. Archival Internships : A Guide for Faculty, Supervisors and Students. Chicago, Society of American Archivists, 2008 ; CHAMPAGNE, 
Michel. « Le rôle stratégique du stage dans la formation en archivistique : réflexion d'un superviseur de stages ». Archives, vol. 42, n² 2, 2010-2011, p. 107-116.

37. - PIFER, Richard L. « [Notes de lecture à propos de] Archival Internships : A Guide for Faculty, Supervisors and Students ». The American Archivist, vol. 71, $\mathrm{n}^{\circ} 2,2008$, p. 556-559.

38. - ROUSSEAU, Jean-Yves. «Étude descriptive de la contribution du stage à la formation professionnelle initiale des archivistes au Québec de 1983 à 1994 ». Archives, vol. 29, nos 3-4, 1997-1998, p. 19-47, p. 27.

39. - Ranim Ayoube, dans le cas qu'elle étudie, évalue le délai nécessaire à cette transformation en deuxième année de master à un mois et demi (La construction de la professionnalité émergente..., thèse cit., p. 89).

40. - CHAMPAGNE, Michel. « Le rôle stratégique du stage... ». Art.cit., p. 107-116.

41. - Ibid., p. 113-114.

42. - En sociologie, l'habitus est « ce qui explique que, placés dans des conditions similaires, les agents aient la même vision du monde, la même idée de ce qui se fait et ne se fait pas, les mêmes critères de choix [...] ». WAGNER, Anne-Catherine. " Habitus ». Dans PAUGAM, Serge (dir.). Les 100 mots de la sociologie. Paris : Presses universitaires de France, coll. «Que sais-je ? », 2010. Selon Bourdieu, inventeur du concept, l'habitus garantit plus sûrement que les normes ou les consignes la conformité des pratiques.

43. - On peut bâtir des indicateurs pour mesurer ces facteurs d'adhésion. À Angers, certaines questions de l'enquête annuelle auprès des étudiants de chaque promotion permettent de les quantifier.

44. - Ont participé à cette recherche collective sous la direction de Patrice Marcilloux, professeur d'archivistique, Noura Bahidj, Baptiste Bonnefoy, Fanny Bourgin, Sébastien Chetanneau, Christine-Rose d'Armagnac de Castanet, Bérangère Esnault, Marion Ferrer, Marine Gaudin, Béatrice Gesland, Marine Kounkou-Kekolo, Camille Nef, Camille Olivier, Natacha Potereau, Sabrina Tavernier.

45. - Enquête administrée par voie électronique entre le 19 et le 27 janvier 2015 auprès de 121 personnes inscrites en master Métiers de l'enseignement, de l'éducation et de la formation (MEEF) documentation ( $1^{\text {re }}$ et $2^{\mathrm{e}}$ années), en licence professionnelle Traitement des archives et des bibliothèques, en master Métiers des archives et des bibliothèques ( $1^{\text {re }}$ et $2^{\mathrm{e}}$ années) à l'université d'Angers, avec un taux de réponse de près de $50 \%$. Voir le site : http://alma.hypotheses.org/1530.

46. - Voir le site : https://www.change.org/p/citoyens-contre-le-projet-de-réglementeuropéen-sur-les-données-personnelles-eudatap [consulté le 27/09/2016]. 47. - Voir le site : http://alma.hypotheses.org/?p=1572 [consulté le 27/09/2016].

\section{RÉSUMÉS}

Entre une formation professionnalisée à l'université et la délimitation d'une profession existent de nombreuses interactions et dépendances. La formation suit les évolutions du métier mais le métier est aussi influencé par l'existence de formations longues et diplômantes qui le légitime et le valorise. Le métier d'archiviste n'échappe pas à ce constat général qui conditionne l'acquisition d'une identité professionnelle par les étudiants. L'assimilation de ce qu'en sciences de l'éducation 
on nomme professionnalité et qui ne recouvre pas que la capacité d'agir avec discernement dans une situation donnée de travail mais intègre aussi la capacité à donner du sens à une activité, à dialoguer avec les représentations, les normes, les valeurs qui se tissent autour de cette activité, est un processus complexe et dynamique qui se traduit par une adhésion et une conformité aux pratiques mais avec un regard distancié, grâce à un processus de mise en savoirs abstraits ou formels. Il en découle une forte interdépendance entre les différents acteurs, chacun concourant à sa place à la reconnaissance et la légitimation de la profession. Une formation universitaire ne peut se développer en décalage avec le milieu professionnel de référence, même si elle se doit d'introduire un point de vue distancié qui est la marque du monde universitaire et un gage d'adaptabilité pour les futurs professionnels, mais une profession a aussi besoin de l'existence de diplômes reconnus et de formalisation des savoirs.

Between a professionalised university training course and the definition of a profession there are of course many interactions and mutual influences. Training must follow the changes in a profession but the profession is also influenced by the availability of longer-term training programmes and training courses which offer qualifications that legitimise and enhance the profession. The profession of archivist is governed by this general educational rule which is at work in the way students acquire their professional identity. The assimilation of what education sciences call 'professionality' does not merely mean the capacity for making informed decisions in a given work situation. It also involves the capacity to give meaning to an activity, to enter into dialogue with the representations, norms and values that surround this activity. This is a complex and dynamic process. It implies an adhesion and conformity with practices, but with a dispassionate view, thanks to a process of mobilisation of abstract and formal knowledge. The result is a strong interdependance between the different actors which contribute to the recognition and legitimation of the profession. A university training course cannot be developed if it is not connceted with the professional milieu it prepares for, even though it must also offer a critical posture, which is the hallmark of a university education and a guarantee of adaptability for future profesionnals. But a profession also requires formal qualifications, recognised diplomas and a formalisation of its skills.

\section{INDEX}

Mots-clés : professionnalité, archiviste, enseignement universitaire

Keywords : professionnality, archivist, university Education

\section{AUTEUR}

\section{BÉNÉDICTE GRAILLES}

Maîtresse de conférences en archivistique, Centre de Recherches Historiques de l'Ouest (CERHIO), UNIV Angers, CNRS, Université Bretagne-Loire benedicte.grailles@univ-angers.fr 